\title{
Incidence and Management of Infusion Reactions to Infliximab in a Prospective Real-world Community Registry
}

\author{
Denis Choquette, Rafat Faraawi, Andrew Chow, Jude Rodrigues, William J. Bensen, \\ and Francois Nantel
}

ABSTRACT. Objective. Infliximab (IFX) is a therapeutic monoclonal antibody targeting tumor necrosis factor- $\alpha$ indicated in the treatment of chronic inflammatory diseases. IFX is administered by intravenous infusion and may be associated with different types of infusion reactions.

Methods. RemiTRAC Infusion (NCT00723905) is a Canadian observational registry in which patients receiving IFX are followed prospectively to document premedication use, adverse events, infusion reactions, and the management of infusion reactions. The primary endpoint was to assess factors associated with infusion reactions.

Results. There were 1632 patients enrolled and 24,852 infusions recorded. Most patients (63.1\%) were treated for rheumatologic conditions such as rheumatoid arthritis, ankylosing spondylitis, or psoriatic arthritis. Of the 1632 patients, 201 (12.3\%) reported at least 1 infusion reaction. Three hundred twenty-two infusions were associated with an infusion reaction (1.3\%), and most were mild to moderate in severity (95\%). The most common infusion reactions were pruritus (19.9\%), flushing $(9.9 \%)$, or dyspnea $(6.2 \%)$. Multivariate analysis showed that antihistamines premedication, number of previous infusion reactions, and female sex were significantly associated with an increased incidence of infusion reactions ( $\mathrm{p}<0.0011$ ). The use of any concomitant immunosuppressant or corticosteroids did not influence the incidence of infusion reactions. Antihistamine premedication was associated with an increased incidence of infusion reactions (OR 1.58, $\mathrm{p}=0.0007$ ).

Conclusion. This registry shows that in community-based infusion clinics, infusion reactions to IFX are uncommon and mild to moderate in nature. Antihistamines, intravenous steroids, and acetaminophen are widely used as preventative premedication, although this study showed an absence of benefit with their use. (First Release June15 2015; J Rheumatol 2015;42:1105-11; doi:10.3899/jrheum.140538)

Key Indexing Terms:

INFLIXIMAB

INTRAVENOUS INFUSION

REGISTRY

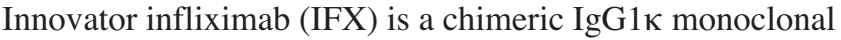
antibody approved for the treatment of inflammatory conditions such as rheumatoid arthritis (RA), ankylosing spondylitis (AS), psoriatic arthritis (PsA), plaque psoriasis (PSO), Crohn disease (CD), and ulcerative colitis (UC) ${ }^{1,2,3,4}$. IFX binds specifically with high affinity to the soluble and transmembrane forms of human tumor necrosis factor- $\alpha$ (TNF- $\alpha$ ) and neutralizes its biological activity.

IFX is administered through an intravenous (IV) infusion

From the Institut de Rhumatologie de Montréal, Montréal, Quebec; McMaster University, Hamilton; Credit Valley Hospital, Mississauga; Clinical Research and Arthritis Centre, Windsor; Janssen Inc., Toronto, Ontario, Canada.

Study supported and conducted by Janssen Inc.

D. Choquette, MD, Institut de Rhumatologie de Montréal; R. Faraawi, MD, McMaster University; A. Chow, MD, Credit Valley Hospital;

J. Rodrigues, MD, Clinical Research and Arthritis Centre; W.J.Bensen, MD, McMaster University; F. Nantel, PhD, Janssen Inc.

Address correspondence to Dr. F. Nantel, Janssen Inc., 19 Green Belt Drive, Toronto, Ontario M3C 1L9, Canada.E-mail: fnantel@its.jnj.com

Full Release Article. For details see Reprints/Permissions at jrheum.org Accepted for publication March 26, 2015. over a period of $2 \mathrm{~h}$. Patients treated with IFX usually receive induction dosing with administration at a dose of $3-5 \mathrm{mg} / \mathrm{kg}$ at weeks 0,2 , and 6 , followed by regularly scheduled maintenance therapy every $6-8$ weeks. One of the most common types of adverse reactions associated with IFX are infusion reactions, which are reported to occur in about $4 \%$ of infusions in clinical trials. In Canada, IFX is predominantly administered in community clinics using a standardized protocol based on a previous study ${ }^{5}$. Several studies have shown that IFX infusions are safe in this community setting, with a low incidence of infusion reactions $6,7,8,9,10$. However, a large prospective study powered to investigate strategies to minimize the incidence and examine outcomes of these infusion reactions was needed.

\section{MATERIALS AND METHODS}

Study design. RemiTRAC (Remicade Treatment Registry Across Canada) Infusion (NCT00723905) was a multicenter, prospective, observational registry designed to collect and analyze infusion-related real-world data in patients treated with IFX at 12 sites across Canada from August 2005 to October 2012. RemiTRAC Infusion was conducted according to the Declaration of Helsinki. All patients provided informed written consent. The

Personal non-commercial use only. The Journal of Rheumatology Copyright @ 2015 . All rights reserved. 
study protocol was reviewed and approved by the appropriate institutional review board (IRB Services, Canada) for nonacademic sites or respective Research Ethics Boards at each academic investigational site.

Patient population. To be eligible for inclusion in RemiTRAC Infusion, patients had to be a candidate to receive IFX as per the Canadian Product Monograph, provide informed consent, be prescribed IFX by an appropriate physician, and receive the IFX infusions in a community-based infusion center. Patients could enter the registry at any point during their treatment with IFX. All analyses were carried out for 3 different groups - the total population, IFX-naive, and IFX-experienced cohorts - to identify any trends and potential biases.

Data collection. Data was collected at baseline and at each infusion visit. Baseline data included medical history, indication for IFX therapy, and previous (past 3 yrs) and concomitant medication. Data collected at every scheduled infusion visit included medication history, adverse events (AE) since last visit, serious $\mathrm{AE}$, and $\mathrm{AE}$ during infusion (infusion reaction). Also collected were IFX dose, duration of infusion, medication administered before an infusion to prevent infusion reactions (including over-the-counter or prescription prophylaxis taken by the patient before the infusion), and the outcome of the infusion (completed, stopped and restarted, stopped and not restarted). An infusion reaction was defined as any AE occurring during the infusion or within $24 \mathrm{~h}$ postinfusion. Physicians classified the intensity of all infusion reactions as mild, moderate or severe, or serious or not serious. Healthcare professional intervention, any alteration in the infusion procedure, the administration of medication, and the final outcome of the infusion reaction were also recorded.

AE were either reported by the subject voluntarily or were obtained by means of interviewing subjects at study visits. All AE were described and recorded on the subject's source document and case report form including date of onset, seriousness, severity, outcome, action taken, and causal relationship as evaluated by the investigator. All AE were followed to satisfactory resolution or a clinically stable endpoint.

Statistical methods. The primary endpoint was to explore variables associated with the incidence of infusion reactions. Major secondary endpoints were to evaluate the effect of preinfusion medications and concomitant medications on infusion reactions, to describe postinfusion reaction management approaches, and to describe safety variables for patients exposed to IFX.

Multilevel logistic regression analysis was conducted to identify factors associated with the incidence of infusion reactions. The first step performed was the bivariate test of association to identify which factors were associated with the incidence of infusion reactions. During the second step, factors associated with the incidence of infusion reactions at a significance level of $20 \%$ in the previous step were entered in a multivariate multilevel logistic regression to determine which were still associated to the incidence of infusion reactions while controlling for other covariates.

Logistic regression analysis was conducted to compare the incidence of infusion reactions between infusions with pretreatment and infusions where no pretreatment was used. Also, binary variables were derived to indicate which type of concomitant medications was used at time of infusion. The groups of concomitant medications of interest were any immunosuppressant (IS), methotrexate (MTX), azathioprine (AZA), 6-mercaptopurine (6-MP), and corticosteroids. The generalized linear mixed model (SAS GLIMMIX Procedure) was used to take account of the correlation among data from the same subject across consecutive infusion administration visits. Because of the observational nature of our study, the effect was estimated using propensity score risk adjustment with the following variables as predictors: patient age, weight, sex, prior enrollment use of IFX, any prior biologic use, and disease indication for the use of IFX. The predictors also included the following infusion characteristics: the season of infusion, the year of infusion, patient eligibility for the infusion, the IFX dose, the time since the last infusion, and number of previous infusion reactions. A patient randomeffect was included in the model to account for the correlation among data from the same patient across consecutive infusion administration visits.

\section{RESULTS}

The patients' baseline characteristics are presented in Table 1. A total of 1632 patients were recruited for a total followup of 3427.2 patient-years with a mean of 15.3 infusions per patient. The majority of patients in this cohort $(63.1 \%)$ were treated with IFX for rheumatologic conditions such as RA (40.1\%), AS (17.5\%), and PsA (5.5\%). Other patients were treated for PSO (9.4\%), CD (18.8\%), or UC (6.7\%). Thirty-four patients were treated for other indications (juvenile idiopathic arthritis, Behçet disease, uveitis, and other). Those patients were identified as protocol violations. Their data were included in the "total" cohort, but were not presented in more detail. As expected, patients with RA were predominantly women $(72.5 \%)$ while patients with AS were predominantly men (73.8\%). The use of MTX as concomitant medication was more common in patients with RA $(66.8 \%)$ and PsA (58.4\%) while patients with inflammatory bowel disease (IBD) were more likely to receive AZA/6-MP at baseline. Corticosteroid use at baseline varied widely from $5.2 \%$ in patients with PSO to $56.9 \%$ in UC. The registry included both IFX-naive $(\mathrm{n}=1069,65.5 \%)$ and patients with previous infusions of IFX $(\mathrm{n}=563,34.5 \%)$ at entry. In the latter cohort, 205 patients $(13.5 \%)$ had received 1-7 IFX infusions previously while the remaining 358 (21.9\%) received 8 to 82 prior infusions of IFX. A total of 232 patients $(16.7 \%)$ had been previously treated with at least 1 biologic agent other than IFX. The most common previously used biologic agent was etanercept (11.4\%), followed by adalimumab $(6.0 \%)$.

A total of 24,852 IFX infusions were recorded (Table 1). The $\mathrm{n}$ value for infusions peaked at $\mathrm{n}=892$ for infusions numbers 2 and 3, and then decreased to less than 90 for infusion number 45 and above. In the IFX-naive cohort, the maximal observed number of sequential infusions was 50 . Interestingly, there was a large variation in the use of prophylactic premedication between indications (Table 1). Indeed, just over $40 \%$ of infusions administered to patients with a rheumatologic condition (i.e., RA, AS, PsA) were premedicated with the most commonly used agent being acetaminophen. In contrast, premedication was prescribed in about $60-67 \%$ of infusions of patients with IBD and IV steroids were used over $50 \%$ of the time. Patients with PSO were premedicated for $53 \%$ of their infusions. Over the course of the study, 1088 out of 1632 patients discontinued their participation. The reasons for ending participation were treatment completed/receive as planned (35.5\%), disease progression/absence of response (27.1\%), $\mathrm{AE} \mathrm{(11.3 \% ),} \mathrm{and}$ other reasons including loss to followup and withdrawal of consent $(26.1 \%)$. There were 7 fatalities.

A total 322 infusions were associated with an infusion reaction with an overall incidence of $1.3 \%$. There were 201 subjects $(12.3 \%)$ who experienced at least 1 infusion reaction. The number of subjects with at least 1 infusion reaction was higher in the IFX-naive cohort $(13.5 \%$ vs $10.2 \%$ in the

Personal non-commercial use only. The Journal of Rheumatology Copyright $\odot$ (2015. All rights reserved 
Table 1. Subjects' baseline characteristics and infusion outcomes. Values are n (\%) unless otherwise specified.

\begin{tabular}{|c|c|c|c|c|c|c|c|}
\hline Indication & Total & RA & AS & PsA & PSO & $\mathrm{CD}$ & $\mathrm{UC}$ \\
\hline \multicolumn{8}{|l|}{ Baseline characteristics } \\
\hline $\mathrm{n}$ & $1632(100)$ & $654(40.1)$ & $286(17.5)$ & $89(5.5)$ & $153(9.4)$ & $307(18.8)$ & $109(6.7)$ \\
\hline Female & $910(55.8)$ & $474(72.5)$ & $75(26.2)$ & $41(46.1)$ & $67(43.8)$ & $181(59.0)$ & $53(48.6)$ \\
\hline Baseline weight, kg, mean & 78.8 & 77.3 & 80.4 & 86.6 & 93.6 & 72.5 & 76 \\
\hline Remicade-naive & $1069(65.5)$ & $468(71.6)$ & $228(79.7)$ & $62(69.7)$ & $89(58.2)$ & $136(44.3)$ & $63(57.8)$ \\
\hline No. infusions/subject, mean & 15.3 & 17.0 & 16.5 & 13.9 & 13.0 & 13.0 & 11.9 \\
\hline Total exposure, patient-yrs, & n 3427.2 & 1569.6 & 657.8 & 169.1 & 290.7 & 552.6 & 174.4 \\
\hline Exposure, patient-yrs, mean & n $\quad 2.1$ & 2.4 & 2.3 & 1.9 & 1.9 & 1.8 & 1.6 \\
\hline \multicolumn{8}{|l|}{ Concomitant medication } \\
\hline Any IS & $975(59.7)$ & $497(76.0)$ & $114(39.9)$ & $60(67.4)$ & $73(47.7)$ & $154(50.2)$ & $57(52.3)$ \\
\hline MTX & $663(40.6)$ & $437(66.8)$ & $80(28.0)$ & $52(58.4)$ & $38(24.8)$ & $33(10.7)$ & $9(8.3)$ \\
\hline Acetaminophen & $8424(33.9)$ & $3528(31.8)$ & $1681(35.7)$ & $444(35.9)$ & $808(41.0)$ & $1347(34.0)$ & $492(38.0)$ \\
\hline Antihistamines & $5672(22.8)$ & $2378(21.5)$ & $865(18.4)$ & $135(10.9)$ & $557(28.3)$ & $1283(32.3)$ & $342(26.4)$ \\
\hline Steroids & $5587(22.5)$ & $1582(14.3)$ & $783(16.6)$ & $65(5.3)$ & $273(13.9)$ & $2161(54.5)$ & $660(51.0)$ \\
\hline \multicolumn{8}{|l|}{ Infusion reactions } \\
\hline No. infusion reactions & $322(1.3)$ & $182(1.6)$ & $47(1.0)$ & $6(0.5)$ & $27(1.4)$ & $40(1.0)$ & $12(0.9)$ \\
\hline \multicolumn{8}{|l|}{ No. subjects with at least } \\
\hline 1 infusion reaction & $201(12.3)$ & $112(17.1)$ & $25(8.8)$ & $6(6.7)$ & $20(13.2)$ & $25(8.2)$ & $7(6.4)$ \\
\hline
\end{tabular}

RA: rheumatoid arthritis; AS: ankylosing spondylitis; PsA: psoriatic arthritis; PSO: psoriasis; CD: Crohn disease; UC: ulcerative colitis; IS: immunosuppressant; MTX: methotrexate; AZA: azathioprine; 6-MP: 6-mercaptopurine.

IFX-experienced cohort). The mean time to onset of an infusion reaction was $62.4 \pm 60.3 \mathrm{~min}$. Of the 322 infusion reactions recorded, 154 were qualified as mild, 152 were moderate, and $16(5 \%)$ were severe ( 2 were of unknown severity). A total of 26 of those infusion AE led to discontinuation of IFX therapy. There were 4 serious infusion reactions: temperature increase (moderate), itching/flushing (mild, but required hospitalization), chest pain/flushing, and chest pain alone (moderate); the latter 2 occurred in the same patient, but at different times (3-mos apart). All the events occurred during the infusion, with the itching/flushing and chest pain/flushing events occurring rapidly after initiation of infusion (15-29 min). None of the patients discontinued therapy. There was no serious anaphylactic reaction recorded.

An infusion reaction incidence of $1.5 \%$ (228/15242) was observed in the IFX-naive cohort in contrast to $0.98 \%$ (94/9610) in patients who had received previous IFX infusions prior to study entry. The timing of infusion reactions is shown in Figure 1. Interestingly, an infusion reaction incidence of $2.1 \%(22 / 1069)$ was observed at infusion number 1 . The incidence then varied from $1.8 \%$ to $3.2 \%$ between infusions number 2 and 8 , which corresponds roughly to the first year of therapy. Infusions after the first year of treatment were associated with a lower incidence $(<2 \%)$ of infusion reactions. The latest infusion reaction was recorded at infusion number 41 .
The most common infusion $\mathrm{AE}$ was pruritus, which occurred in $19.9 \%$ of infusion reactions (Table 2). AE that occurred in $\geq 4 \%$ of infusion reactions were flushing $(9.9 \%)$, dyspnea $(6.2 \%)$, nausea $(4.7 \%)$, urticaria $(4.7 \%)$, and headaches $(4.0 \%)$.

Table 3 describes the management approach to infusion reactions along with the outcome. Use of medication or an intervention from a healthcare professional occurred in $88.51 \%$ of infusion reactions. Medications, such as antihistamines or steroids, were used in $54.97 \%$ and $22.05 \%$ of infusion reactions, respectively. Epinephrine was used on 2 occasions only. Under most circumstances following the appearance of an infusion reaction, the healthcare professional slowed (36.65\%) and/or stopped (66.77\%) the infusion of IFX and infused normal saline (70.81\%). Both of these strategies appeared successful because the infusion of IFX was restarted in $75.8 \%$ of cases where it had been stopped, and the infusion rate was re-increased in $85.7 \%$ of cases where it had been slowed.

An important question was whether having an infusion reaction increased the risk of having an additional reaction in subsequent infusions. A total $87.6 \%$ of patients $(1425 / 1632)$ never experienced an infusion reaction. Of the 195 patients who experienced an infusion reaction, $60.2 \%$ of them $(121 / 195)$ did not experience any subsequent infusion reactions after a first occurrence. The remaining 74 patients

Personal non-commercial use only. The Journal of Rheumatology Copyright $\odot$ 2015. All rights reserved. 


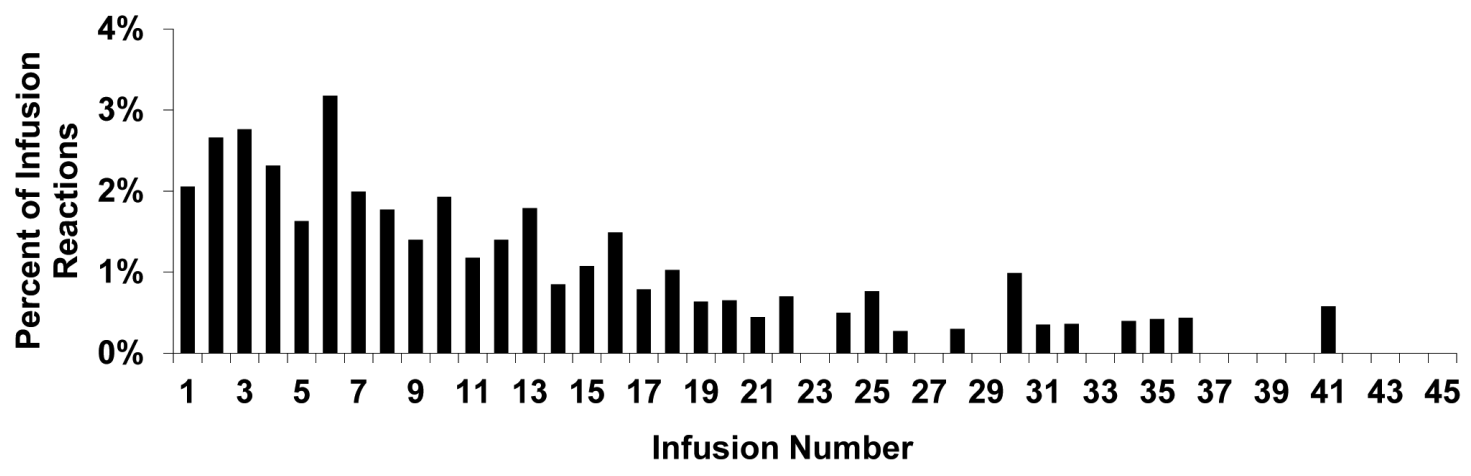

Figure 1. Percentage of infusion reactions per infusion number in all patients.

Table 2. Types of infusion reactions ( $\geq 2 \%$ ).

\begin{tabular}{lcc}
\hline & $\mathrm{n}$ & $\%$ \\
\hline Pruritus & 64 & 19.9 \\
Flushing & 32 & 9.9 \\
Dyspnea & 20 & 6.2 \\
Nausea & 15 & 4.7 \\
Urticaria & 15 & 4.7 \\
Headache & 13 & 4.0 \\
Hypertension & 12 & 3.7 \\
Rash & 11 & 3.4 \\
Chest discomfort & 10 & 3.1 \\
Chest pain & 9 & 2.8 \\
Dizziness & 9 & 2.8 \\
Back pain & 8 & 2.5 \\
Muscle spasms & 8 & 2.5 \\
BP increased & 7 & 2.2 \\
Pruritus, generalized & 7 & 2.2 \\
Other, < $\%$ & 82 & 25.5 \\
\hline
\end{tabular}

BP: blood pressure.

Table 3. Management of infusion reactions.

\begin{tabular}{|c|c|c|c|c|}
\hline \multicolumn{2}{|l|}{ Variables } & \multirow{2}{*}{$\begin{array}{c}\mathrm{n} \\
\\
\\
177\end{array}$} & \multirow{2}{*}{$\begin{array}{c}\begin{array}{c}\text { Infusions } \\
\text { with } \\
\text { Reactions, } \%\end{array} \\
54.97\end{array}$} & \multirow{2}{*}{$\begin{array}{c}\text { All } 24,852 \\
\text { Infusions, \% } \\
0.71\end{array}$} \\
\hline Medications & Antihistamine & & & \\
\hline & Steroids & 71 & 22.05 & 0.29 \\
\hline & Epinephrine & 2 & 0.62 & 0.01 \\
\hline \multirow[t]{9}{*}{ Other } & Oxygen & 16 & 4.97 & 0.06 \\
\hline & $\begin{array}{l}\text { Increased monitoring, } \\
\mathrm{q} \times \min \end{array}$ & 178 & 55.28 & 0.72 \\
\hline & Infusion stopped & 215 & 66.77 & 0.87 \\
\hline & Infusion restarted & 163 & 50.62 & 0.66 \\
\hline & $\begin{array}{l}\text { Infusion slowed } \\
\text { Infusion rate }\end{array}$ & 118 & 36.65 & 0.47 \\
\hline & re-increased & 100 & 31.06 & 0.40 \\
\hline & Saline infused & 228 & 70.81 & 0.92 \\
\hline & Activate EMS & 3 & 0.93 & 0.01 \\
\hline & Other & 1 & 0.31 & 0.00 \\
\hline \multicolumn{2}{|c|}{ Any treatment } & 285 & 88.51 & 1.15 \\
\hline
\end{tabular}

EMS: emergency medical services.
(39.8\%) experienced 1 to 4 additional infusion reactions. The timing of those subsequent infusion reactions is shown in Figure 2. The infusion that immediately followed an infusion reaction was associated with a $33 \%$ incidence of a subsequent infusion reaction (52/158). The risk of subsequent infusion reaction gradually decreased, but still remained elevated compared with patients who never experienced an infusion reaction despite the use of prophylactic premedication in $\sim 75 \%$ of infusions (not shown).

Univariate and multivariate model analyses were used to identify factors associated with the incidence of infusion reactions. From a clinical perspective, we observed that a number of infusion-related variables (concomitant medication, use of antihistamine or steroid premedication, duration of infusion, IFX dose, time since last infusion, number of previous infusion reactions, and even the season) significantly affected the risk of infusion reactions in the univariate model. However, the multivariate model analysis showed that the use of antihistamines as pretreatment $(2.49 \%$ vs $1.32 \%$ if not used, $\mathrm{p}<0.0001)$, the number of previous infusion reactions (OR 2.04, 95\% CI 1.78-2.34, p < 0.0001), the time in days since last infusion (OR $0.99,95 \%$ CI $0.99-1.00$, p < $0.0006)$, female sex $(2.21 \%$ vs $1.49 \%$ for males, $\mathrm{p}<0.0001)$, and the use of corticosteroids at enrollment in patients with previous infusions with IFX ( $2.98 \%$ vs $1.20 \%$, p < 0.002$)$ were significantly associated with an increased incidence of infusion reactions. In contrast, the incidence of infusion reaction decreased with each passing year (OR $0.68,95 \% \mathrm{CI}$ $0.63-0.73, \mathrm{p}<0.0001)$.

A propensity scores-adjusted effect analysis was done to further analyze the effect of pretreatment medication on the incidence of infusion reactions because multiple combinations of these medications could be used (Table 4). In the absence of pretreatment and the presence of any pretreatment, there were $1.14 \%$ and $1.47 \%$ infusion reactions, respectively, a difference that was not statistically significant $(\mathrm{p}=0.1491)$. However, there were significant differences when specific pretreatments were compared. There was a significantly higher rate of infusion reactions compared to no treatment

Personal non-commercial use only. The Journal of Rheumatology Copyright @ 2015 . All rights reserved. 


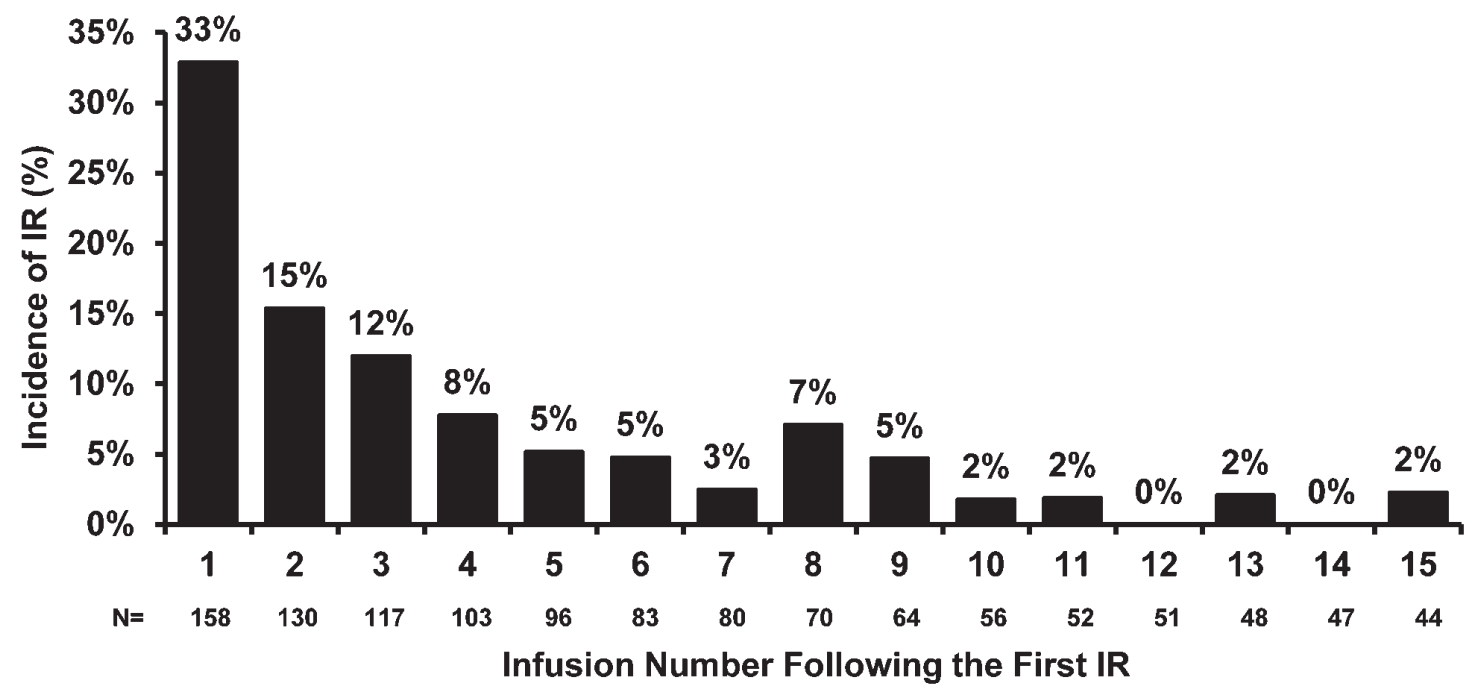

Figure 2. Incidence of infusion reactions (IR) following the first IR.

Table 4. Effect of concomitant medication and premedication on the incidence of infusion reactions (propensity scores adjusted effect).

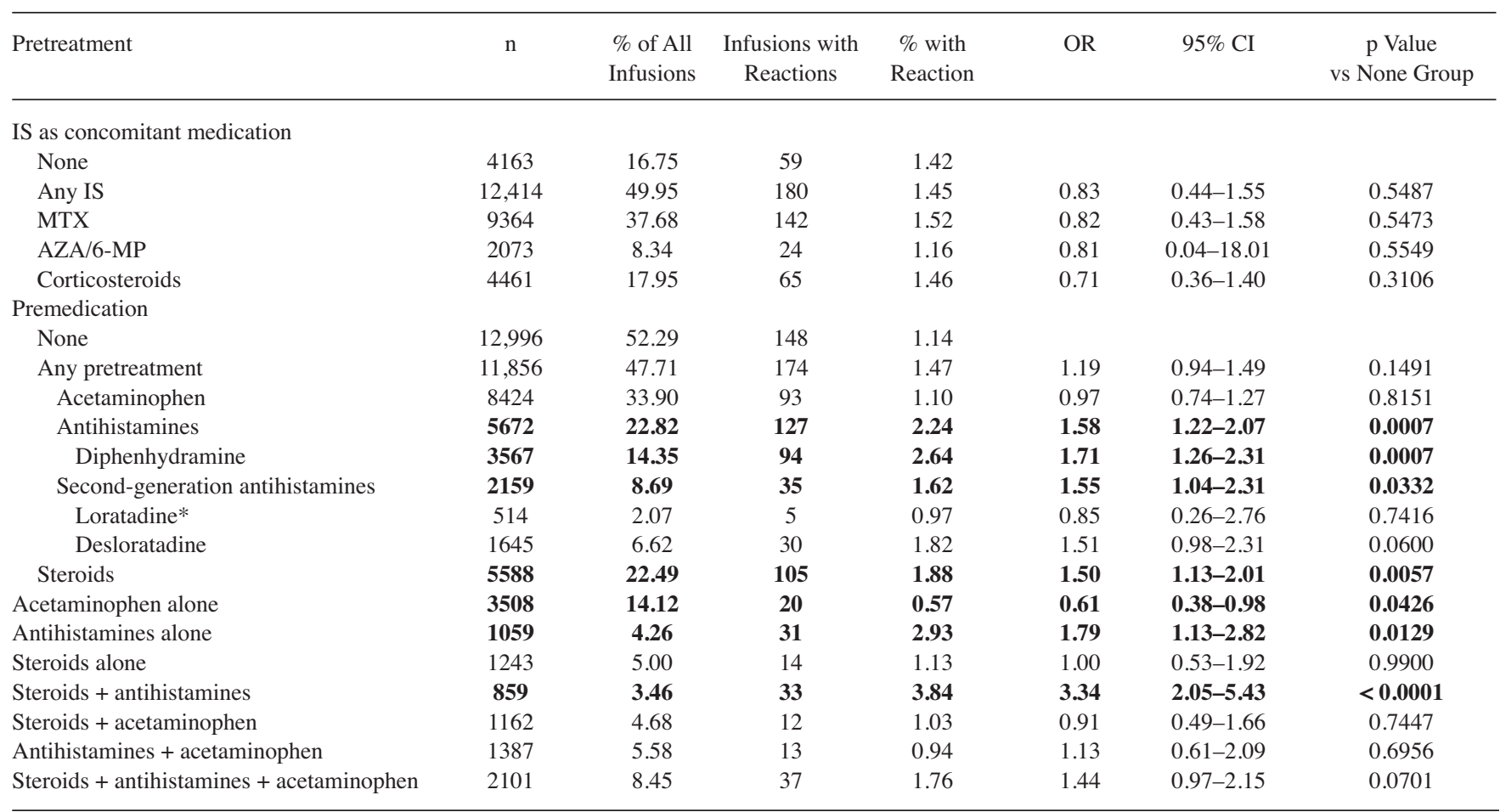

* Because of the lack of statistical power, propensity scores adjustment could not be performed for loratadine. Significant data are in bold face. IS: immunosuppressant; MTX: methotrexate; AZA: azathioprine; 6-MP: 6-mercaptopurine.

when antihistamines $(\mathrm{p}=0.0007)$ and steroids $(\mathrm{p}=0.0057)$ as a group were used in pretreatment. Pretreatment with the first generation antihistamine diphenhydramine significantly increased the odds of an adverse infusion reaction (OR 1.71, $p=0.0007)$. Steroids alone had no significant effect on infusion reactions (OR 1.00, p $=0.9900)$ and it was only when they were used with antihistamines that the risk of a reaction increased compared with no pretreatment (OR 3.34, $\mathrm{p}<0.0001)$. Antihistamines alone significantly increased the risk of infusion reactions (OR 1.79, $\mathrm{p}=0.0129)$. In contrast, acetaminophen alone decreased the risk of infusion reactions (OR 0.61, $\mathrm{p}=0.0426$ ). This finding was consistent with a previously published cohort ${ }^{7}$. The effect of concomitant medication (MTX, AZA/6-MP, corticosteroids) on infusion

Personal non-commercial use only. The Journal of Rheumatology Copyright @ 2015 . All rights reserved. 
reactions was similarly analyzed, but none had any significant effect (data not shown).

\section{DISCUSSION}

RemiTRAC Infusion represents the largest prospective cohort of IFX infusions in a community setting. The incidence of infusion reactions observed in this registry $(1.3 \%)$ was actually lower than the $2.5-6.1 \%$ incidence reported in other cohort studies $5,6,7,8,9$. One reason may be that RemiTRAC Infusion is a longterm registry that was ongoing for 7 years. Our data show that about two-thirds of infusion reactions were observed during the first year of therapy and the mean number of infusions per patients was about 15, representing over 2 years of exposure. Other studies, and all randomized controlled trials, had a much shorter observation period ${ }^{5,6,7,8,9}$. Most centers in our study had large patient populations. It is speculated that the level of confidence from experienced personnel can reflect on the patients and lower their anxiety. Indeed, the reduction of infusion reactions over time could also reflect improved experience of the sites or maybe less frequent reporting of insignificant infusion reactions. Patients were being infused following a standardized infusion protocol that was based on Cheifetz, et al's study ${ }^{5}$, and it was already observed that such a protocol was associated with low incidences of infusion reactions ${ }^{6}$. The majority of patients received IFX as an induction dosing followed by regularly scheduled maintenance therapy, a strategy that has been shown to maximize clinical response and minimize the development of antibodies to IFX. About one-third of the cohort had received previous infusions of IFX prior to their recruitment in the study. Therefore, there was the potential for an exclusion bias where patients who had experienced an infusion reaction in the past were more likely to drop out of therapy and less likely to be recruited.

A large number of infusion-related factors were found to affect the risk of infusion reaction. Many of those factors were found to be associated to biases that are inherent to observational noninterventional studies. However, the prospective nature of this registry and its size did allow for multivariate models and propensity score-adjusted analyses. The positive association with the number of previous infusion reactions makes logical and clinical sense. The year of administration is likely a surrogate of the site's experience and comfort level with the infusions. The use of glucocorticoid at study entry in patients already receiving IFX may be associated with disease severity or a partial response to IFX, both of which could lead a higher risk of AE. Factors such as the infusion duration and the time since the last infusion were found to be statistically significant, but likely clinically irrelevant because their effect sizes were very small. The higher incidence in women is noteworthy and likely contributes to the variability between indications.

We did not observe any effect of concomitant medication on the incidence of infusion reactions. Our data differ from those of Lee, et al ${ }^{10}$, who reported a statistically significant decrease in the incidence of infusion reaction in patients under concomitant IS in this IBD-enriched cohort. Our data did show a small negative trend in patients treated with AZA/6-MP.

In this registry, we observed differences in the use of concomitant medication and premedication between indications. We did not observe that the use of either antihistamines or IV steroids as premedication decreased the incidence of infusion reactions. Indeed, we observed a higher incidence of infusion reactions with antihistamines and/or steroids compared with patients who did not receive any premedication, similar to findings published by Wasserman, et al ${ }^{7}$. This is most likely the result of a selection bias whereby patients who had a history of infusion reactions were thus more likely to have a subsequent one and to receive premedication. Under such a premise, however, the lower incidence of infusion reactions with acetaminophen alone is interesting and warrants further investigation. The effect of acetaminophen on infusion reactions was previously reported in a retrospective chart review of 447 patients with $\mathrm{CD}$ with over 6468 IFX infusions in which infusions premedicated with acetaminophen were associated with the lowest incidence of infusion reactions ${ }^{9}$. The mechanism of action of acetaminophen suggests that most acute infusion reactions might be noninflammatory and nonimmunologically mediated in nature. This was suggested by Cheifetz, et $a l^{5}$, who reported that infusion reactions were not associated with elevated immunoglobulin E or the release of mast cell tryptase, which are more characteristic of type 1 hypersensitivity reactions. The current data raise the question as to whether antihistamines and IV steroids have any benefit in preventing infusion reactions. There were 2 placebo (PBO)-controlled trials that evaluated premedication strategies to prevent the incidence of infusion reaction using IV steroids. The most commonly cited study is from Farrell, et $a l^{11}$, in which 80 patients with $\mathrm{CD}$ were randomized to IV hydrocortisone $200 \mathrm{mg}$ or PBO immediately before their first and subsequent IFX infusions. They reported that the hydrocortisone-treated patients had a lower incidence of antibodies to IFX and infusion reactions compared with the patients who received PBO. However, this difference was not statistically significant for infusion reactions $(p=0.15)$ and the patients in this trial were all receiving episodic/on-demand IFX therapy, a treatment strategy that is known to be associated with the development of high levels of immunogenicity as evidenced by the extremely high incidence of infusion reactions in this trial ( $24 \%$ in the PBO group compared with $1.3 \%$ in our study). Still, the conclusions from our study likely explain the high usage of IV steroids among gastroenterologists, especially considering that "on-demand/episodic" dosing for IFX was the standard treatment regimen for $\mathrm{CD}$ until the publication of the ACCENT I trial (A Crohn's Disease Clinical Trial

Personal non-commercial use only. The Journal of Rheumatology Copyright @ 2015 . All rights reserved. 
Evaluating Infliximab in a New Long-term Treatment Regimen) in $2006^{12}$. The trial by Sany, et $a l^{13}$ was a large double-blind 36-week trial in which 355 patients with RA were premedicated with either $\mathrm{PBO}$ or betamethasone $(0.5$ $\mathrm{mg} / \mathrm{kg} \mathrm{IV}$ ) prior to their IFX infusion. In this trial, the incidence of infusion reaction was actually greater in the betamethasone group compared with PBO $(5.0 \%$ vs $2.5 \%$, respectively, $\mathrm{p}=0.05$ ). The result from this trial could be interpreted as more clinically meaningful than the Farrell, et al study because the patients in the Sany, et al cohort received IFX as induction and maintenance therapy, which is currently the most common strategy for treatment with IFX. Finally, Lee, et al did not observe any effect of corticosteroid premedication in their RA cohort ${ }^{10}$.

There are several limitations to our study. The most important is the risk for selection biases that are inherent in noninterventional, observational studies, although we attempted to limit them using multivariate and propensity score analyses. Also, by definition, reactions to the infused product were included if they occurred within $24 \mathrm{~h}$. Thus, any delayed reactions, such as a skin rash occurring 24-72 h following exposure, would most likely be missed in this analysis.

The RemiTRAC Infusion registry demonstrates that, in real-world practice, infusion reactions to IFX are rare, mild to moderate in nature, and successfully managed by the healthcare professionals in place using a standard infusion protocol ${ }^{8,10}$. We did not observe any particular strategy to decrease the incidence of infusion reaction, although the use of acetaminophen may be beneficial when used alone. After reviewing the data, there was a consensus among the investigators that, considering the low incidence of infusion reaction, no premedication is recommended. Once an infusion reaction has occurred, premedication could be considered and tailored to the type of infusion reaction prior to the next infusion.

\section{ACKNOWLEDGMENT}

The authors acknowledge the following individuals for their help during the course of this study: John Leombruno (Schering-Plough) for his input in the original design; Patricia Bandeira, Saliha Boumaza (Schering-Plough/ Merck), Faye Cross, and Debra Mitchell (Janssen) for operations; Merlin Njoya (McKesson) for statistical analyses; Vincent Letourneau, Warren J. Winkelman, May Shawi, Allen Lehman, and Susan Otawa (Janssen) for manuscript review and discussions; and finally, all the remaining RemiTRAC Infusion investigators: Frédérique Morin, Carter Thorne, Wayne Gulliver, Richard Langley, Tim McCarthy, John Watterson, and Mahjed Kraishi.

\section{REFERENCES}

1. Silva LC, Ortigosa LC, Benard G. Anti-TNF-a agents in the treatment of immune-mediated inflammatory diseases: mechanisms of action and pitfalls. Immunotherapy 2010;2:817-33.

2. Wiedmann MW, Mössner J, Baerwald C, Pierer M. TNF alpha inhibition as treatment modality for certain rheumatologic and gastrointestinal diseases. Endocr Metab Immune Disord Drug Targets 2009;9:295-314.

3. Feldmann M, Maini SR. Role of cytokines in rheumatoid arthritis an education in pathophysiology and therapeutics. Immunol Rev 2008;223:7-19.

4. Smolen JS, Emery P. Infliximab: 12 years of experience. Arthritis Res Ther 2011;13 Suppl 1:S2.

5. Cheifetz A, Smedley M, Martin S, Reiter M, Leone G, Mayer L, et al. The incidence and management of infusion reactions to infliximab: a large center experience. Am J Gastroenterol 2003;98:1315-24.

6. Ducharme J, Pelletier C, Zacharias R. The safety of infliximab infusions in the community setting. Can J Gastroenterol 2010;24:307-11.

7. Wasserman MJ, Weber DA, Guthrie JA, Bykerk VP, Lee P, Keystone EC. Infusion-related reactions to infliximab in patients with rheumatoid arthritis in a clinical practice setting: relationship to dose, antihistamine pretreatment, and infusion number. J Rheumatol 2004;31:1912-7.

8. Kelsall J, Rogers P, Galindo G, De Vera MA. Safety of infliximab treatment in patients with rheumatoid arthritis in a real-world clinical setting: description and evaluation of infusion reactions. J Rheumatol 2012;39:1539-45.

9. Keshavarzian A, Mayer L, Salzberg B, Garone M, Finkelstein W, Cappa J, et al. A multicenter retrospective experience of infliximab in Crohn's disease patients: infusion reaction rates and treatment persistency. Gastroenterol Hepatol 2007;3:381-90.

10. Lee TW, Singh R, Fedorak RN. A one-hour infusion of infliximab during maintenance therapy is safe and well tolerated: a prospective cohort study. Aliment Pharmacol Ther 2011;34:181-7.

11. Farrell RJ, Alsahli M, Jeen YT, Falchuk KR, Peppercorn MA, Michetti P. Intravenous hydrocortisone premedication reduces antibodies to infliximab in Crohn's disease: a randomized controlled trial. Gastroenterology 2003;124:917-24.

12. Rutgeerts P, Diamond RH, Bala M, Olson A, Lichtenstein GR, Bao $\mathrm{W}$, et al. Scheduled maintenance treatment with infliximab is superior to episodic treatment for the healing of mucosal ulceration associated with Crohn's disease. Gastrointest Endosc 2006; 63:433-42.

13. Sany J, Kaiser MJ, Jorgensen C, Trape G. Study of the tolerance of infliximab infusions with or without betamethasone premedication in patients with active rheumatoid arthritis. Ann Rheum Dis 2005;64:1647-9. 